\title{
Capacidad de carga turística como herramienta para el desarrollo sostenible de playas: Caso Montañita, provincia de Santa Elena, Ecuador
}

\section{Tourist load capacity as tool for sustainable development of beaches: Montañita case, province of Santa Elena, Ecuador}

\author{
Paola Cecilia Gálvez-Izquieta'; Arnaldo Efrén Mendoza-Tarabó \\ 1 Universidad Católica de Santiago de Guayaquil, Ecuador. \\ 2 Universidad Estatal Península de Santa Elena, Ecuador.
}

\section{Resumen}

El presente estudio evidencia la disposición de una herramienta de organización turística que permita realizar acciones a partir de la estimación de la carga turística de la playa de Montañita para el desarrollo sostenible de la misma, dado que es necesario afrontar su excesiva afluencia de visitantes como una problemática latente. Con dicha intención, no solo se recabaron datos bibliográficos y estudios relevantes a la problemática, sino también las impresiones en cuanto al grado de satisfacción alcanzado por los visitantes del destino expuesta a través de la escala de Likert. Los conceptos mencionados permiten evidenciar las posibilidades de mejora de la playa de Montañita con la correcta organización y diligencia municipal, así como el soporte de profesionales del área turística, gracias a que el abordaje del tema presenta una somera crítica de la situación actual que evidenciar el caso de estudio, su alcance y consecuencias, así como la falta de aplicación de la herramienta en mención.

Palabras clave: capacidad de carga turística; planificación; carga turística; desarrollo sostenible.

\begin{abstract}
The present study evidences the disposition of a tool for tourist organization that allows to carry out actions from the estimation of the tourist load of the beach of Montañita for the sustainable development. With this intention, bibliographic data and studies relevant to the problem were collected, but also the impressions regarding the degree of satisfaction achieved by the visitors of the destination exposed through the Likert scale. The aforementioned concepts make it possible to demonstrate the possibilities of improving Montañita beach with the correct organization and municipal diligence, as well as the support of professionals from the tourist area, and the approach to the topic presents a brief critique of the current situation that evidences the case study, its scope and consequences, as well as the lack of application of the tourism carrying capacity as tool.
\end{abstract}

Keywords: tourist load capacity; planning; tourism load; sustainable development.

Paola Gálvez-Izquieta

Universidad Católica de Santiago de Guayaquil, Av. Carlos Julio Arosemena, km. 1.5

e-mail: paola.galvez@cu.ucsg.edu.ec

Recibido: Nov 28, 2019 Aprobado: Ene 14, 2020

doi: $10.23878 /$ empr.v14i1.171
Cómo citar:

Gálvez-Izquieta, P., \& Mendoza-Tarabó, A. (2020). Capacidad de carga turística como herramienta para el desarrollo sostenible de playas: Caso Montañita, provincia de Santa Elena, Ecuador. Revista Empresarial, 14(1), 1-7. 


\section{INTRODUCCIÓN}

La motivación del presente estudio hace referencia al análisis de la capacidad de carga turística que suele ser empleado como un instrumento de planificación y gestión de acciones que aporten de forma significativa al desarrollo sostenible del turismo y cuidado del entorno, motivo por el que el caso de estudio evalúa cuán efectivo es emplear la técnica en mención, contemplando diversas variables (Tarlombani da Silveira, 2015).

Se ha tenido en consideración que uno de los principales desafíos en términos de ordenamiento para el municipio, la junta parroquial y la comuna de las áreas costeras (así como las demás instituciones públicas y privadas estrechamente ligadas al rubro), es precautelar que el desarrollo de las actividades turísticas sea ejecutado con un precedente sostenible sin ocasionar afectaciones al entorno y fomentar la viralización de prácticas social y ambientalmente responsables entre las entidades gubernamentales y la colectividad (Andrade, Arenas, \& Guijón, 2018).

El caso expuesto concierne a la comuna de Montañita, zona geográficamente rodeada de cerros y flora al pie del mar que está localizada a 180 kilómetros de la ciudad de Guayaquil y es reconocida como una de las playas ecuatorianas con mayor auge turístico por la formación de olas cuya altura promedio es de 2.5 metros en sus costas, atractivo que la convierte en el destino predilecto para prácticas y campeonatos de surf, ecuavolley y demás actividades deportivas o de esparcimiento, en donde los turistas extranjeros contemplen un espacio que les permita tener libertad de ideologías y a la vez, mantener contacto con la naturaleza.

Cabe mencionar que el adecuado ordenamiento de los destinos permitirá también planificar y ejecutar estrategias que impulsen el desarrollo económico de la zona, ejecutando acciones que oferten una atención mayormente homogénea a los visitantes sin generar impactos negativos (Ochoa, 2014).

Sustentando dicha postura, el estudio pretende evidenciar la utilidad de determinar la capacidad de carga en las playas como Montañita, con la finalidad de proteger el entorno del ecosistema del destino -en la medida de lo posible- y disminuir aquellas afectaciones suscitadas por la afluencia de visitantes y la generación descomunal de desechos en las costas, arista desde la que se extiende un aporte teórico y la exposición de los criterios pertinentes, así como la relevancia que tiene incentivar el levantamiento de investigaciones similares (Evert, 2001).

Cabe mencionar la consideración y relevancia de estudios previos en los que se consideró en primera instancia la determinación del perfil de los visitantes de Montañita, así como sus motivaciones para conocer el destino y cuán satisfechos estuvieron con la travesía, evidenciando en un caso particular, que el principal detonante para elegir dicho punto, es disfrutar de la playa, permitiendo así que "prestadores de servicios turísticos diseñen productos y ofrezcan servicios acorde a la demanda" (Carvache, Wilmer, \& Mónica, 2017).

Por otra parte, los precedentes del destino en cuanto a ordenamiento territorial para la comunidad representan el incontrolable crecimiento de la afluencia turística en la que los comuneros han adoptado plazas de trabajo, pero no han precautelado la conservación responsable del espacio y sus recursos, lo cual evidencia la imperativa necesidad del ordenamiento de actividades que se desarrollan permitiendo el mantenimiento adecuado del medio ambiente. (Ortega, 2010)

La estructura del documento contempla la fundamentación teórica de los lineamientos requeridos para el cálculo de la capacidad de carga turística y el desarrollo sostenible enfocado al turismo, lo cual expone la viabilidad de la playa de Montañita como destino; posteriormente, el análisis de la incidencia de los indicadores socioculturales y ambientales, como conclusión (Echamendi, 2001). 


\section{MÉTODO}

Tras considerar el precedente territorial, se procede a analizar la capacidad de carga turística en la playa de Montañita para su desarrollo sostenible, y posteriormente se plantean los objetivos específicos: levantar una línea base sobre el estado actual de la playa de la comuna Montañita en relación con la aplicación de la herramienta de capacidad de carga; identificar el grado de satisfacción de los usuarios de la playa de la comuna Montañita y, proponer los lineamientos para la aplicación de la capacidad de carga para el desarrollo sostenible de la playa de Montañita, provincia de Santa Elena, Ecuador (González \& Carmona, 2015).

El modelo de investigación aplicada es de índole descriptivo, empleado con la intención de generar una base que permita analizar datos y posteriormente organizar dicha información para esclarecer las particularidades del objeto de estudio.

Las técnicas de investigación aplicadas se han sustentado de la revisión bibliográfica de instrumentos documentales, de alcance descriptivo con enfoque correlacional y la correspondiente asociación de variables; la población objeto de estudio fue determinada por los turistas que visitaron la playa de Montañita durante el mes de julio del año en curso, empleado por entrevistadores debidamente entrenados, con una duración aproximada de 8 minutos por participante. Independiente a la triangulación de la información documental recabada, el interés del proceso investigativo recae en la percepción de los protagonistas del caso, profesionales del sector turístico y representantes comunales implicados.

Para los fines pertinentes, se realizó el cálculo del tamaño muestral aleatorio con la aplicación de la siguiente fórmula en donde el intervalo de confianza empleado fue del $95 \%$ y una correspondencia de heterogeneidad del $5 \%$ :

$$
n=\frac{N}{1+\frac{e^{2}(N-1)}{z^{2} p q}}
$$

En referencia a la acotación del Ministerio de Turismo (2018), el temporal de carnaval del período en mención en la provincia de Santa Elena contó con el ingreso de alrededor de 301000 turistas, lo cual corresponde al resultado de una muestra de 384 personas encuestadas.

\section{RESULTADOS}

Los estímulos provocados en los viajeros al momento de llegar a un nuevo destino, desencadena un sinnúmero de impresiones que no son siempre consideradas, pese a que dicha información puede ser transformada en oportunidades de mejora y de nueva evaluación para el destino. Con dicho precedente, la autora aplicó una encuesta descriptiva con preguntas cerradas con la finalidad de determinar la percepción de los turistas que visitan la playa de Montañita, misma que fue valorada a través de la escala de Likert, método de medición que permite evaluar el comportamiento de las personas (Carvache, Torres, \& Carvache, 2017, págs. 113 - 129).

Un $48 \%$ la total insatisfacción expresada por los turistas encuestados en la playa de Montañita, respecto a los servicios de playa en general. Si bien es cierto, parte de los participantes empatizaron al respecto (14\% y 15\%) correspondientemente, pero la inclinación mayoritaria, fue negativa (ver Figura 1).

Se evidenció que el $55 \%$ de los turistas encuestados está totalmente insatisfecho con el proceso de limpieza de la zona de arena en la playa de Montañita exponiendo que la misma cuenta con muchos desperdicios en su superficie. El 12\% expresó su parcial satisfacción; el 10\% parcial insatisfacción y el 23\% total satisfacción. El 38\% de la población sujeto de estudio, está satisfecha con la calidad del agua en Montañita. Sin embargo, cabe mencionar que el $26 \%$ rechaza dicha afirmación (ver Figura 2). 


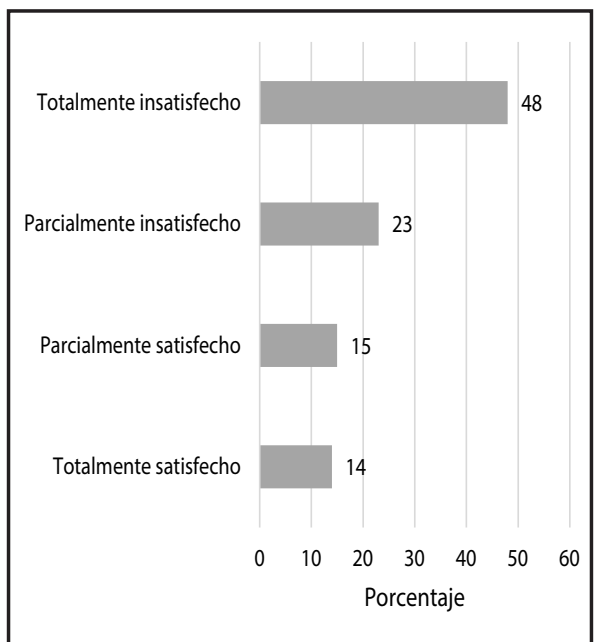

Figura 1. Satisfacción general de servicios en la playa

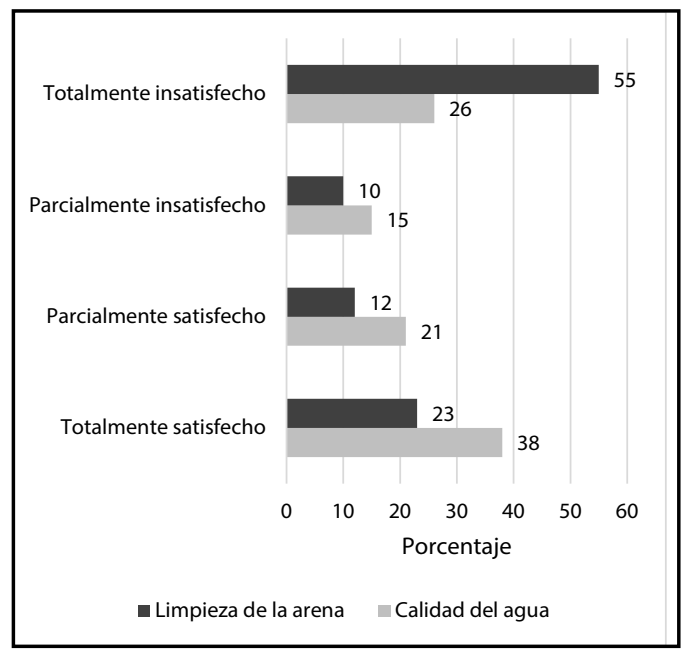

Figura 2. Satisfacción con los recursos naturales de la playa

Se observaron muy bajos porcentajes de incidencia positiva respecto a la disposición de baños públicos, destacando que el $63 \%$ de los participantes indicó su total insatisfacción por la ausencia de baterías sanitarias de libre acceso. El 68\% de los turistas que visitan Montañita, evidenció su total insatisfacción respecto a la ausencia de duchas públicas. Dado es el escenario que en caso de tratarse de una visita corta al destino, los viajeros no pueden disponer de duchas a menos que contaran con un servicio de hotel reservado. Por otro lado, se corroboró una falta de papeleras o contenedores selectivos de desechos por la incidencia en un $51 \%$ de total insatisfacción expresada por los encuestados (ver Figura 3).

En cuanto al servicio de parqueos, el $61 \%$ de los encuestados está totalmente insatisfecho, situación que se suscita por la desorganización de la zona dispuesta para dicho fin y el entorpecimiento vial en la zona próxima a la playa suscitada por la desinformación de los turistas (Osorio, 2006). Se observó alto nivel de complacencia de los turistas, representado por el $39 \%$ de total satisfacción respecto a las señaléticas y paneles de información dispuestos en la playa de Montañita. Pese a ello, es válido mencionar que el $26 \%$ y $19 \%$ se mostró parcial y totalmente insatisfecho al respecto.

El 32\% de los encuestados está totalmente satisfecho con el servicio de comunicación (megafonía), dispuesto en la playa de Montañita, seguido por el 27\% de parcial insatisfacción dado que dicha herramienta comunicativa no cuenta con el alcance requerido.Finalmente, la última consulta realizada a los turistas sostiene su insatisfacción total, respecto a la disposición de personal y equipos correspondientes para situaciones de riesgo por la ausencia de un servicio de salvamento y primeros auxilios (Figura 4).

\section{DISCUSIÓN}

La necesidad de calcular la capacidad de carga turística de la comuna Montañita evidencia la ausencia de un proceso de ordenamiento territorial del espacio en que se desenvuelven las actividades de esparcimiento y ocio de forma continua, considerando la importancia de no generar perjuicios al medio ambiente y a su vez, contribuir con el impulso de acciones de turismo sostenible (Boullon, 2015).

En base a la argumentación de que la capacidad de carga establece el número de personas que tolera una zona geográfica puntual sin causar impactos ambientales al área o afectaciones a los comuneros, es válido analizar que el término de turismo sostenible está directamente relacionado al determinar que la intención en sí es fomentar el máximo aprovechamiento de recursos del destino (sociales, económicos, culturales y ambientales) sin afectar el nivel de satisfacción de los turistas u originar impactos negativos en el entorno, especialmente al contemplar la trascendencia de establecer no sólo el auge de visitantes máximo permitido, sino también normar el tipo de actividades y comportamientos que presenta la población flotante que visita estos espacios (Picornell, 2015). 


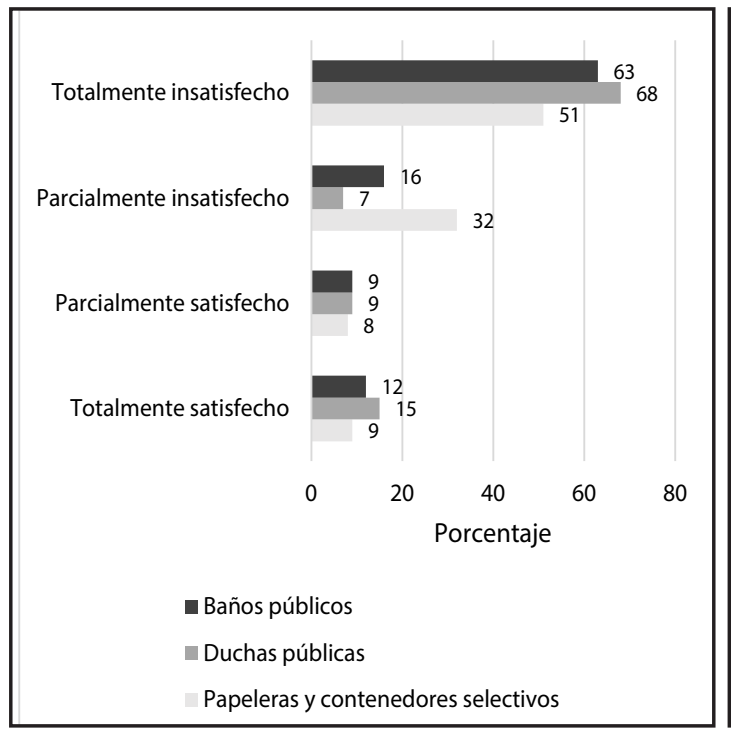

Figura 3. Satisfacción con servicios de aseo

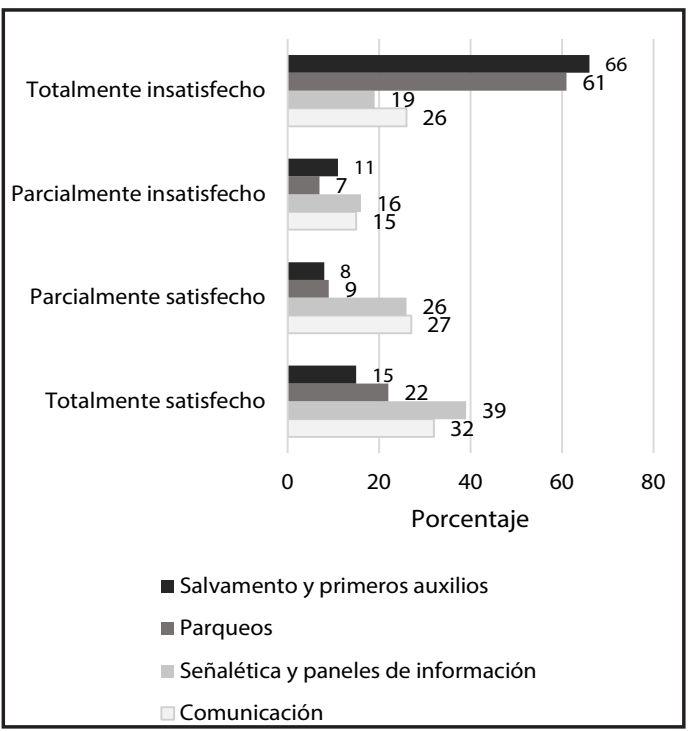

Figura 4. Satisfacción con otros servicios

En términos turísticos, la posición que ocupan los atractivos turísticos en una zona tiene una estructura natural inherente a partir del cual se debe plantear su desenvolvimiento empleando la carga turística como herramienta para desarrollar la zona de manera sostenible, añadiendo de forma medible los elementos que requiera el caso, sin que se entorpezca el entorno o se ocasione afectaciones para con los comuneros; es decir, que el escenario idóneo sería responder a los requerimientos del espacio de forma orgánica (Castro \& Morales, 2006).

Desde este punto, se conoce que el exceso de intercesión humana, causará afectaciones extremadamente perjudiciales para el destino y/o sus pobladores, por lo que la determinación de límites juega un papel fundamental dado que establecerá una alerta para que los recursos del destino fueran explotados de forma responsable (Opaschowski, 2015).

Es entonces, que el caso específico de la comuna de Montañita muestra que se continúa admitiendo una excesiva cantidad de visitantes que permanecen en la zona (cifra que incrementa en las épocas de alta estacionalidad como carnaval, semana santa y las festividades de diciembre, etc.), escenario en el que los hoteles y restaurantes no se dan abasto de forma adecuada, fomentando el uso de la herramienta de capacidad de carga, dado que indicaría la incapacidad de la comuna para recibir mayor cantidad de paseantes, ya que en sus inicios no fue diseñada ni planificada con fines turísticos, sino residenciales.

La zona de uso turístico de la playa de Montañita cuenta actualmente con una capacidad física de 1200 personas, índice que representa el tope máximo de visitantes que potencialmente podría acudir de forma simultánea al destino con sus requerimientos cubiertos satisfactoriamente. Con este precedente, la intención de cubrir las necesidades de la comuna resultaría inoperante ya que la suficiencia de un factor no puede compensar la falta de otro, debido a que cada aspecto responde a las diversas necesidades de los visitantes. Por esta razón, la capacidad global de recepción de usuarios en la playa está definida por la menor de las capacidades analizadas (Korstanje, 2012).

En cuanto a la oferta existente en hospedaje y alimentación se rescata que la misma es superior a la capacidad física de la zona de uso turístico de la playa, lo cual no necesariamente promueve un exceso de visitantes en la zona de playa, ya que Montañita posee una vida turística muy activa en el centro de la población, y los turistas que hacen uso de los servicios de alojamiento y alimentación no acuden a la playa al unísono (Blanco, 2014).

Por otra parte, la prevalencia de hospedaje no reglado es una muestra de la creciente demanda por plazas de alojamiento (inclusive por una oferta económica más accesible), evidenciando que un incremento de dicha índole debe planificarse y monitorearse, de 
modo que no crezca de forma desproporcionada y no promueva un exceso de turistas a los que no se debería admitir (Monge \& Yagué, 2016).

Así mismo, se debe tener en cuenta cualquier consideración referente a ampliaciones de infraestructuras, misma que aplica solamente en el caso hipotético de que la capacidad de manejo de usuarios dado por los servicios existentes fuese igual a la capacidad física de la playa, pero la realidad muestra que actualmente ni los servicios ni el personal encargado de seguridad son suficientes para recibir el número de visitantes que la playa físicamente podría acoger.

Existe una alta capacidad de manejo actualmente en la comuna de Montañita, sin embargo, es válido acotar que no es factible incrementar la capacidad turística mientras no sea considerado el aumento de servicios básicos de la demanda actual, misma que supera la población local. Es decir, que la capacidad de la naturaleza de absorber los impactos causados ha sido sobrepasada, dado que incluso en términos socioculturales se ha desplazado a la población local desencadenando una problemática aguda por el uso de los espacios (Ministerio del Ambiente, 2014).

\section{CONCLUSIONES}

En referencia a la conceptualización y aplicación de la herramienta de capacidad de carga turística, se evidencia que la misma puede ser efectivamente útil en casos de ecosistemas no intervenidos con sus correspondientes poblaciones animales (para lo cual fue creada en primera instancia) y, al trasladarse a una realidad con injerencia humana, la adopción del instrumento se dificulta ya que el comportamiento de las personas ejerce diversas reacciones y consecuencias que no pueden ser totalmente arbitradas, escenario al que se suman variables como las preferencias y arraigos culturales de la población y la forma en la que la actividad turística como tal se desarrolla.

El espacio físico de la playa se ha visto disminuido por la dinámica evolutiva del perfil costanero, es decir que existe una reducción en la zona de arena propiamente con el simultáneo incremento de la capacidad efectiva. Pese a ello sigue existiendo una debilidad en la dotación de servicios básicos, detonando el problema de alcantarillo que causa un gran impacto ambiental con altos picos de sobrecarga durante los fines de semana y feriados, siendo el de carnaval el más destacado por el número de turistas que saturan la localidad.

Los estudios ejecutados en diversos destinos turísticos han permitido detectar que las limitantes conforme a la afluencia de visitantes no es la única variable que causa afectación, dado que también están en consideración los propios planes y metas de expansión y desarrollo de un área determinada, en donde se verán directamente afectados los niveles de calidad y de vida en la zona, motivo por el que las consideraciones respecto al crecimiento del espacio en cuanto al sistema turístico, son inminentes.

La mayoría de afectaciones evidenciadas en las áreas turísticas, no son consecuencia del número de personas admitidas en la zona, sino más bien, resultante del tipo de comportamiento que tienen los turistas, mismos que deben ser normados previo a la consideración de limitar la afluencia de visitantes.

\section{REFERENCIAS}

Andrade, B., Arenas, F., \& Guijón, R. (2018). Revisión crítica del marco institucional y legal chileno de ordenamiento territorial: el caso de la zona costera. Santiago: Instituto de Geografía - Pontificia Universidad Católica de Chile.

Blanco, F. (2014). Reflexiones sobre seguridad, poderes públicos y actividad turistica. Madrid: Instituto de Estudios Turísticos - Secretaría General de Turismo.

Boullon, R. (2015). Planificación del Espacio Turístico - 7ma Edición. México: Trillas.

Carvache, W., Torres, M., \& Carvache, M. (2017). Análisis del perfil y satisfacción del turista que visita Montañita - Ecuador. Guayaquil: Revistas Científicas, Universidad de Murcia / Escuela Superior Politécnica del Litoral, ESPOL.

Castro, C., \& Morales, E. (2006). La zona costera. Medio natural y ordenación integrada. Santiago: Revista de Geografía Norte Grande - Serie GEOlibros N 5 . 
Cendrero, A. (1997). Indicadores de desarrollo sostenible para la toma de decisiones. Cantabria: Universidad de Cantabria.Cervantes, J. (2007). El ordenamiento territorial como eje de planeación de proyectos de turismo sustentable. Viña del Mar: Ciencias Sociales Revista Electrónica, Universidad de Viña del Mar.

Cordero, E. (2011). Ordenamiento territorial, justicia ambiental y zonas costeras. Valparaíso: Revista de Derecho de la Pontificia Universidad Católica de Valparaíso.

Echamendi, P. (2001). La capacidad de carga turística. Aspectos conceptuales y normas de aplicación. Anales de Geografía de la Universidad Complutense, Madrid.

Egio, C., \& Torrejón, E. (2015). Identidad, reconocimiento y participación. Ordenamiento territorial y justicia ambiental en las zonas rurales de Medellín (Colombia). Medellín: Universidad de Antioquia.

Evert, K.-J. (2001). Paisaje y urbanismo: léxico multilingüe de planificación, diseño, y protección del medio ambiente. Berlín.

González, L., \& Carmona, M. (2015). Guía para la medición directa de la satisfacción de los clientes. Andalucía: IAT - Instituto Andaluz de Tecnología.

Hervé, D. (2010). Notion and elements of environmental justice: guidelines for its implementation in territorial planning and strategic environmental evaluation. Santiago de Chile: Universidad Diego Portales.

Korstanje, M. (2012). ¿Se puede ponderar la seguridad turística? Un ensayo conceptual. Curitiba: Universidade Federal do Paraná - Repositorio Digital Institucional.

Martinez, C., \& Rentería, F. (2018). Construcción de puntos ecológicos y disminución de residuos a través de la implementación del reciclaje y la educación ambiental, en la Institución Educativa José Hilario López, Inspección El Triunfo, Municipio de La Montañita - Caquetá. Universidad Nacional Abierta y a Distancia UNAD.

Martínez, J. (2008). Conflictos ecológicos y justicia ambiental. México D.F.: Universidad de Monterrey.

Ministerio del Ambiente, R. O. (2014). Plan de manejo de las áreas protegidas de Galápagos para el Buen Vivir. Galápagos, Ecuador.

Monge, J., \& Yagué, R. (2016). El desarrollo Turístico sostenible - Tren crucero del Ecuador. Buenos Aires: Centro de Investigaciones y estudiso turísticos.

Ochoa, E. (2014). Elaboración de agendas de manejo costero integrado como herramientas complementarias de los PDOT. Manta: Secretaría Técnica del Mar - SETEMAR.

Opaschowski, o. (2015). Turismo de masas o turismo a medida. Límites ecológicos, psicológicos, ecológicos. Hamburgo: Institute for Leisure Research de Hamburgo.

Ortega, M. (2010). Plan de ordenamiento territorial turístico para la comunidad de Montalita, Provincia de Santa Elena, para el fomento del tursimo en la zona. Quito: Universidad Tecnológica Equinoccial.

Ortiz, D. (2016). El turismo masivo y su impacto sociocultural en la comuna Montañita parroquia Manglaralto, provincia de Santa Elena. Guayaquil: Universidad Católica de Santiago de Guayaquil.

Osorio, M. (2006). La planificación turística. Enfoques y modelos. Toluco: Quivera.

Picornell, C. (2015). Los impactos del turismo. Palma de Mallorca: Universitat de Ies Illes Balears.

Picornell, C. (2015). Los impactos del turismo. Palma de Mallorca: Universitat de Ies Illes Balears.

Quintero, J. (2014). Sectores económicos del cantón playas y sus vínculos con el turismo. Madrid: Universidad de la Rioja.

Tarlombani da Silveira, M. (2015). Turismo y sustentabilidad - Entre el discurso y la acción. Curitiba: Universidad Federal de Paraná.

Yepes, V. (1999). LAs playas en la gestión sostenible del Litoral. Valencia: Agencia Valenciana de Turismo. 